\title{
Ruptured ovarian teratoma with granulomatous peritonitis
}

WL Wong *, Anthony WT Chin, WM Yu, FH Ng

Hong Kong Med J 2019;25:490.e1-2

https://doi.org/10.12809/hkmj187710

\section{Case}

In January 2017, a 35-year-old woman was admitted to our hospital with insidious onset of upper abdominal pain. A computed tomography (CT) scan showed bilateral ovarian cysts with fat fluid level, calcifications, and Rokitansky protuberance, compatible with ovarian teratoma (Fig 1). Antidependent fatty pockets with soft tissue rim were found at the bilateral subphrenic space, likely representing reactive changes to spilt cyst content, which also explained the patient's upper abdominal pain. The patient had stable vital signs and was therefore treated conservatively. Followup ultrasonography scan showed globular fatty locules on the liver surface, compatible with escaped fatty cyst content (Fig 2). Subsequently, the patient underwent bilateral ovarian cystectomy. Histology confirmed bilateral ovarian mature cystic teratoma. Intra-operatively, widespread flimsy adhesions and multiple sebum-like implants were seen in the peritoneal cavity, consistent with changes related to teratoma rupture. The peritoneal cavity was irrigated and her symptoms gradually subsided; however, follow-up CT showed mild interval enlargement of the fat-attenuated lesions (Fig 3).

\section{Discussion}

Mature cystic teratomas (also known as dermoid cysts) are common ovarian germ cell neoplasms accounting up to $10 \%$ to $25 \%$ of all ovarian neoplasms. ${ }^{1}$ They are cystic tumours composed of well-differentiated derivations from at least two of the three germ cell layers. Tumours are bilateral in about $10 \%$ of cases. On ultrasonography, cystic teratoma commonly manifests as a cystic lesion with a densely echogenic tubercle projecting into the cystic lumen; or a diffusely or partially echogenic mass with posterior attenuation by sebaceous material and
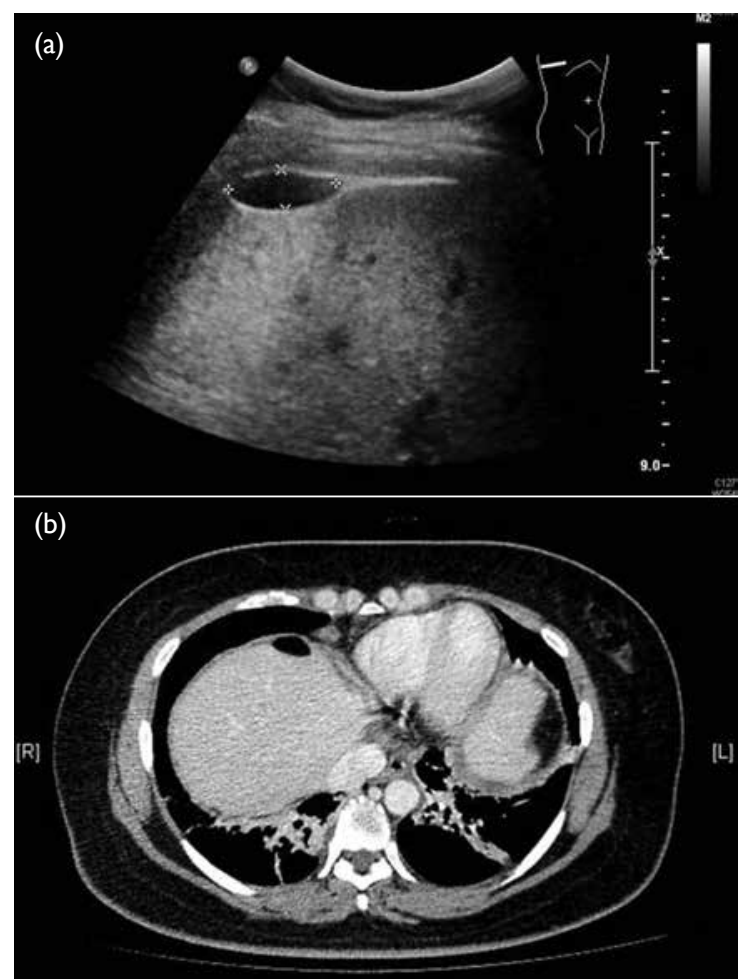

FIG 2. (a) Ultrasonograph showing a hypoechoic lesion and (b) computed tomography image showing a fat-attenuated nodule over the liver surface suggestive of liver capsular implant from ruptured ovarian teratoma partly fat-attenuated mass with tooth like calcification, characteristic of ovarian teratoma 


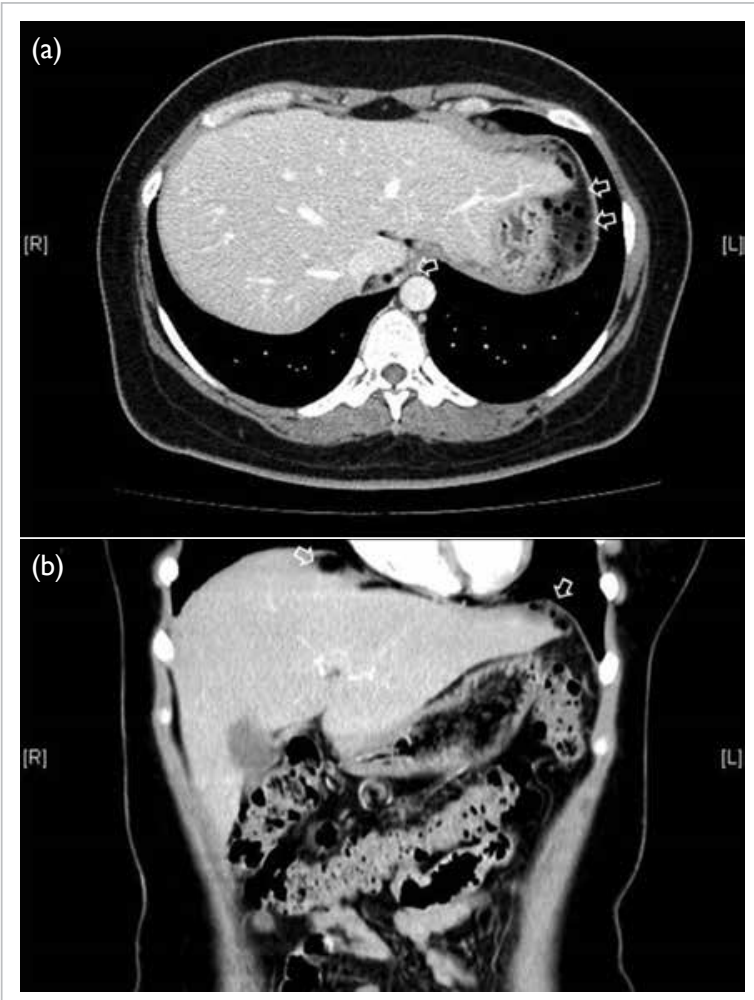

FIG 3. (a) Axial and (b) coronal computed tomography images showing progression of the bilateral subphrenic fatty implants (arrows) with increased adjacent stranding suggestive of chronic granulomatous peritonitis caused by the content of the mature cystic teratoma

hair. Multiple thin echogenic bands caused by hair in cyst cavity can also been seen. Pure sebum within the cyst can be hypoechoic or anechoic, fluid-fluid level can result from sebum floating on aqueous fluid which appears more echogenic than the sebum layer. On CT, the diagnosis of mature cystic teratoma is rather straightforward; fat attenuation within a cyst is diagnostic of mature cystic teratoma. Teeth or other calcifications can be seen in $56 \%$ of cases. ${ }^{2}$

Spontaneous rupture is an uncommon complication of dermoid cysts owing to the presence of a thick capsule, and is only seen in $1 \%$ to $4 \%$ of cases. ${ }^{1}$ Acute peritonitis can result from sudden rupture of tumour contents as seen in the present case. Chronic granulomatous peritonitis is caused by chronically leaking teratoma and is characterised by multiple small white peritoneal implants and dense adhesions with variable ascites. Visualisation of fatty implants within the peritoneal cavity is diagnostic. ${ }^{3}$

The reported CT findings of granulomatous peritonitis or intraperitoneal rupture of teratoma are inconsistent. ${ }^{1,3}$ In one case, capsular fatty implants were seen in the dome of the liver, similar to the CT appearance in the present case. In other patients there can be a significant amount of intraperitoneal free fluid and an omental cake appearance mimicking peritoneal carcinomatosis.

Chronic granulomatous peritonitis is a potentially serious complication of ruptured dermoid cyst and can lead to bowel obstruction resulting from adhesion. Removal of cystic content and copious peritoneal lavage should be performed to prevent new adhesion and peritoneal granuloma, and can be a successful method for treating chemical peritonitis caused by ruptured ovarian teratoma. ${ }^{4}$ In the present case, progression of the right subphrenic fatty implant, possibly related to incomplete removal of the cystic content, resulted in chronic granulomatous peritonitis. The patient may have benefitted from further peritoneal lavage.

\section{Author contributions}

WL Wong contributed to acquisition of data. All authors contributed to concept or design, analysis or interpretation of data, drafting of the article, and critical revision for important intellectual content. All authors had full access to the data, contributed to the study, approved the final version for publication, and take responsibility for its accuracy and integrity.

\section{Conflicts of interest}

All authors have disclosed no conflicts of interest.

\section{Funding/support}

This research received no specific grant from any funding agency in the public, commercial, or not-for-profit sectors.

\section{Ethics approval}

This study was approved by the Kowloon Central/Kowloon East Research Ethics Committee (Ref KC/KE-19-0158/ER-4). Informed consent was obtained from the patient.

${ }^{1}$ WL Wong *, MB, BS, FRCR

${ }^{1}$ AWT Chin, MB, ChB, FRCR

${ }^{1}$ WM Yu, MB, ChB

${ }^{2} \mathrm{FH} \mathrm{Ng}$, FRCR, FHKAM (Radiology)

\section{${ }^{1}$ Department of Radiology, United Christian Hospital, Kwun Tong, Hong} Kong

2 Department of Radiology, Caritas Medical Centre, Shamshuipo, Hong Kong

*Corresponding author: jesswong723@gmail.com

\section{References}

1. Erbay G. Ruptured ovarian dermoid cyst mimicking peritoneal carcinomatosis: CT and MRI. J Clin Anal Med 2016;6:701-3.

2. Outwater EK, Siegelman ES, Hunt JL. Ovarian teratomas: tumor types and imaging characteristics. Radiographics 2001;21:475-90.

3. Fibus TF. Intraperitoneal rupture of a benign cystic ovarian teratoma: findings at CT and MR imaging. AJR Am J Roentgenol 2000;174:261-2.

4. Shamshirsaz AA, Shamshirsaz AA, Vibhaka JL, Broadwell C, Van Voorhis BJ. Laparoscopic management of chemical peritonitis caused by dermoid cyst spillage. JSLS 2011;15:403-5. 